\title{
Welfare Analysis of Tax and Expenditure Reform
}

\author{
Alberto Pench ${ }^{1}$
}

Received: 15 January 2015 / Accepted: 6 July 2015 / Published online: 16 July 2015

(C) Società Italiana degli Economisti (Italian Economic Association) 2015

\begin{abstract}
The motivation of the present paper originates from the current debt crisis of so many developed countries and analyzes, within a simplified model with a single representative consumer, the welfare effect of a combined reduction in indirect taxes and public expenditure. As already demonstrated by a pioneering work by Guesnerie (Econometrica 47:393-421, 1977) and subsequent literature, the bulk of information needed to be sure to get a welfare gain from this reform is heavy; under this respect a feature of the paper, to which less attention was paid, is a more detailed description on the side of public expenditure where multiple expenditure categories are allowed: this feature can be exploited to properly tailor expenditure and tax cuts. The main policy prescription arising from the paper is to concentrate expenditure cuts on categories with close private substitutes and a low marginal willingness to pay for them, and tax decreases on private goods with larger own price effects, weak relations with other private goods and complementary to labour. An important implication is that marginal willingness to pay could be the rationale for a new, or parallel, classification of public expenditure categories carrying incentives toward quality and efficiency.
\end{abstract}

Keywords Tax reform - Public expenditure - Willingness to pay

JEL Classification $\mathrm{H} 21 \cdot \mathrm{H} 50$

I wish to thank a referee for helpful comments.

$\bowtie$ Alberto Pench

alberto.pench@unipi.it

1 Department of Political Sciences, Univeristy of Pisa, Via Serafini 3, 56126 Pisa, Italy 


\section{Introduction}

Tax reform literature addressed a number of issues of theoretical and practical relevance along different lines of research: some works concerned single consumer economies thus focusing on efficiency issues while others introduced distributional considerations employing social welfare functions. From a practical point of view debated issues were the non optimality of an equiproportional taxation and the information requirements needed to implement a Pareto improving tax reform starting from an arbitrarily given tax structure. To cite only some works within the above mentioned streams of research the reader is referred to the works of Diewert (1978), Atkinson and Stiglitz (1980), Corlett and Hague (1953-1954), Yitzhaki and Thirsk (1990), Hatta (1977, 1986) and Ahmad and Stern (1984). Most of these papers neglected the use of tax revenue to finance public expenditure since they considered the substitution of one or more taxes with another or others or a compensating change in lump sum transfers. Notable exceptions were Guesnerie $(1977,1995)$ which explicitly considered a compensating change in public expenditure: a feature of such contributions is that the author focused on the problem of existence and on theoretical analysis of alternative tax and public good equilibria and explicitly denied any interest in canonical formulae for Pareto improving tax reforms expressed in terms of elasticities or other parameters. Other papers dealing with tax and expenditure reforms are found within the vast literature on marginal cost of public funds: Schöb (1994) and Slemrod and Yitzhaki (2001), to cite only two and, more recently, the exhaustive work by Anderson and Martin (2011) to which the reader is referred for detailed references.

Within such a vast literature it is hard to find a gap to be filled and the present paper can be considered a reformulation and extension of some of the results presented in Anderson and Martin (2011) with a deeper emphasis on the side of public expenditure: the aim, in the tradition of tax reform literature, is to find conditions for a Pareto improving tax and expenditure reform in terms of complementarity and substitutability relations among private and public goods and labour but also to point out the characteristics public expenditure categories should possess to achieve such a result. In this respect the paper might be helpful in the difficult choice by the policy maker about where to concentrate expenditure cuts.

The paper includes two sections which present, respectively, a basic version of the model and its generalization with several categories of public expenditure; a final section summarizes the results and briefly comments upon them.

\section{Basic Model}

The economy consists of a single aggregate consumer, $\mathrm{N}$ private goods, a single public good, provided free of charge, and labour. Taxes are levied on private goods and labour and finance public expenditure. Constant return to scale technologies, resulting in fixed producer prices, are assumed for sake of simplicity. Preferences of the representative consumer are described by a well behaved utility function:

$$
U(\mathbf{x}, L, G)
$$


where $\mathbf{x}$ is the vector demand for private goods, $L$ labour supply and $G$ the public good; specific taxes, indicated by $\mathbf{t}$ and $t_{\mathrm{L}}$, are levied on private goods and labour so that consumer prices are $\mathbf{q}=\mathbf{p}+\mathbf{t}$ and gross wage $w=w_{N}+t_{L}$. The choice problem, with budget constraint $q \mathbf{x}=w_{N} L$, generates indirect utility function $V\left(\mathbf{q}, w_{N}, G\right)$. The public budget constraint is:

$$
\mathbf{t x}+t_{L} L=G
$$

where the unit production cost of the public good is normalized to one. This framework serves to model a balanced-budget tax and expenditure reform, where only indirect taxes are concerned on the public revenue side; the motivation of this particular choice is twofold: on the one hand, in a real world context, a reform concentrated only on indirect taxes may be easier to implement than one involving direct taxes as well. On the other hand, in developed countries at least, direct taxes are much more complicated than indirect ones so that $t_{L}$ should be properly interpreted as an average unit tax on labour that summarizes a plurality of tax rates and tax expenditures: this might raise problems as to the interpretation of the tax reduction modelled in the sequel.

The effect of the reform, following the methodology adopted by Auerbach (1985), is found by total differentiation of the indirect utility function and of the public budget:

$$
\begin{aligned}
& d V=-\lambda \mathbf{x d t}+\lambda^{G} d G \\
& d G=\mathbf{t d x}+\mathbf{x d t}+t_{L} d L
\end{aligned}
$$

where $\mathbf{d t}=\left(d t_{1}, \ldots, d t_{N}\right)$ while $\lambda$ and $\lambda^{G}$ are, respectively, the marginal utility of income and the marginal utility of public good. By substitution we get:

$$
d V=-\lambda \mathbf{x d t}+\lambda^{G}\left(\mathbf{t d x}+\mathbf{x d t}+t_{L} d L\right)=\left(\lambda^{G}-\lambda\right) \mathbf{x d t}+\lambda^{G}\left(\mathbf{t d x}+t_{L} d L\right)
$$

The last term in parenthesis can be expressed as:

$$
\left(\mathbf{t N}+t_{L} \mathbf{L}_{\mathbf{i}}\right) \mathbf{d t}+\left(\mathbf{t x}_{\mathbf{G}}+t_{L} \frac{\partial L}{\partial G}\right) d G
$$

where $\mathbf{N}$ is the $\mathbf{N} \times \mathbf{N}$ matrix of price effect whose representative element is:

$$
N_{i j}=\frac{\partial x_{i}}{\partial q_{j}}
$$

$\mathbf{x}_{G}$ is the vector of "expenditure effects" on private goods:

$$
\mathbf{x}_{\mathbf{G}}=\left(\frac{\partial x_{1}}{\partial G}, \ldots, \frac{\partial x_{N}}{\partial G}\right)
$$


while $\mathbf{L}_{i}$ is the vector of price effect on labour supply:

$$
\mathbf{L}_{\mathbf{i}}=\left(\frac{\partial L}{\partial q_{1}}, \ldots, \frac{\partial L}{\partial q_{N}}\right)
$$

Substituting $d G$ from (3) into (4) and collecting terms yields:

$$
d V=\left\{\left(\lambda^{G}-\lambda\right) \mathbf{x}+\lambda_{G} \frac{\left(\mathbf{t N}+t_{L} \mathbf{L}_{\mathbf{i}}\right)+\left(\mathbf{t x}_{\mathbf{G}}+t_{L} \frac{\partial L}{\partial G}\right) \mathbf{x}}{\left(1-\mathbf{t x}_{\mathbf{G}}-t_{L} \frac{\partial L}{\partial G}\right)}\right\} \mathbf{d t}
$$

Using the Slutsky decomposition the term $\left(\mathbf{t} \mathbf{N}+t_{L} \mathbf{L}_{i}\right)$ can be reformulated to give (see Auerbach 1985, p. 120):

$$
d V=\left\{\left(\lambda^{G}-\lambda\right) \mathbf{x}+\lambda_{G} \frac{\mathbf{t} \mathbf{S}+t_{L} \mathbf{L}_{i}^{C}+\left(\mathbf{t x}_{\mathbf{G}}+t_{L} \frac{\partial L}{\partial G}-\mathbf{t x}_{R}-t_{L} \frac{\partial L}{\partial R}\right) \mathbf{x}}{\left(1-\mathbf{t x}_{\mathbf{G}}-t_{L} \frac{\partial L}{\partial G}\right)}\right\} \mathbf{d t}
$$

where $\mathbf{S}$ is the $\mathbf{N} \times \mathbf{N}$ matrix of compensated effect whose representative element is:

$$
S_{i j}=\frac{\partial h_{i}}{\partial q_{j}}
$$

$\mathbf{x}_{R}$ is the vector of income effects on private goods:

$$
\mathbf{x}_{R}=\left(\frac{\partial x_{1}}{\partial R}, \ldots, \frac{\partial x_{N}}{\partial R}\right)
$$

and $\mathbf{L}_{\mathbf{i}}^{C}$ is the vector of price effect on compensated labour supply:

$$
\mathbf{L}_{\mathbf{i}}^{C}=\left(\frac{\partial L^{C}}{\partial q_{1}}, \ldots, \frac{\partial L^{C}}{\partial q_{N}}\right)
$$

To ease the subsequent discussion it is better to rewrite the above expression as:

$$
d V=\left\{A \mathbf{x}+\lambda_{G} \frac{B+(D-C) \mathbf{x}}{1-D}\right\} \mathbf{d t}
$$

It seems natural to ask what expression (7) suggests when a public expenditure cut is backed by a reduction in all tax rates. This means being able to find sufficient (but non necessary) conditions, under which such reform is surely Pareto improving, besides the trivial case when $\lambda^{G}=0$. To simplify matters we concentrate on conditions assuring that both terms in curly brackets are negative.

The first term $(A \mathbf{x})$ is negative, being $\mathbf{x}$ strictly positive, if $\lambda^{G}<\lambda$ : without any further qualification, $\lambda^{G}$ can be greater or smaller than $\lambda$; nevertheless in a real-world setting the initial situation is likely to be far away from an optimum so that, given that $G$ represents total current public expenditure, it is quite reasonable, that its marginal 
utility is positive but less than marginal utility of income. However this condition alone is not sufficient to ensure a Pareto improvement even in the simplest case where a reduction in public expenditure is compensated by an increase in lump sum transfer: in any case vector $\mathbf{x}$ affects the magnitude of the welfare effect.

The second term combines substitution, income and expenditure effects: $B$ is negative if, loosely speaking, compensated own-price effects for taxed goods and (net) complementarity relations with other taxed goods and labour dominate (net) substitutability relations. $C$ is greater than $D$ if positive income effects of the tax cut on the demand for taxed private goods and labour supply dominate negative ones and are greater than the corresponding expenditure effects. Nothing can be said a priori on the sign of $B+(D-C) \mathbf{x}$ but some qualifications emerge from the budget constraint of the representative consumer. It is easy to see that the following restrictions hold:

$$
\mathbf{q} \mathbf{x}_{R}=w_{N} \frac{\partial L}{\partial R}+1 \text { and } \mathbf{q} \mathbf{x}_{G}=w_{N} \frac{\partial L}{\partial G}
$$

Hence:

$$
D-C=w\left(\frac{\partial L}{\partial G}-\frac{\partial L}{\partial R}\right)+\mathbf{p}\left(\mathbf{x}_{R}-\mathbf{x}_{G}\right)-1
$$

The first term in parenthesis on the right hand side is plausibly negative recalling that $\mathrm{G}$ is total public expenditure and its effect on labour supply is the aggregation of (possibly) positive and negative effects determined by specific categories of public expenditure which might partially compensate each other in the aggregate; the second term is likely to be positive given that not all private goods can be inferior and that expenditure effects can be mixed in sign according to substitutability and complementarity relations among $G$ and private goods.

If $B$ and $D-C$ are negative, the second term in curly brackets of (7) is negative if the denominator is positive: this will be the case if negative expenditure effects are predominant among taxed goods; a fortiori the denominator is positive if more heavily taxed goods show a negative expenditure effect. The only restriction imposed upon the sign of such effects is shown in (8): remembering that, within this model, $G$ is total public expenditure, it might be reasonable to assume that expenditure effects are not so large, hence a positive sign for the denominator (and a small magnitude) might be more plausible than a negative one: deeper reflections on the sign of the denominator are deferred to the next section.

Putting together the above remarks we can draw as a general conclusion that to be sure that a proposed tax and expenditure cut is Pareto improving it is crucial to have reliable information about price, income and expenditure effects of the demand for private goods and labour supply and about the marginal utility of public expenditure (or marginal willingness to pay for it): information requirements that are identical to those found by Guesnerie (1995) and surely heavy. But the emphasis of the present paper is on operational guidelines for the policy maker (if any) and in this light it should be noticed that the sign of $d V$ combines the above conditions with vector dt on which there are some degrees of freedom. For example if it is known that the conditions 
for a positive denominator of the second term in (7) are met, then tax cuts should be concentrated on goods with strong compensated own price effects, small (positive) cross price effects on other goods and complementary to labour. A shortcoming of this approach is that once the choice of $\mathbf{d t}$ has been made the government has no control over the resulting $d G$ and this may be a problem if the reform aims at a targeted reduction in public expenditure, as normally happens.

It should be stressed that if there is evidence that positive expenditure effects are strong and predominant this must be carefully taken into account before opting for tax cuts since the sign of the second term in curly brackets of (7) could be reversed. The main conclusion of this section is that the welfare effect of the reform is largely determined by parameters which might be known but not controlled by the government. The extension of the model described in the next section reveals that additional degrees of freedom originate from a more detailed description on the public expenditure side.

Before turning to the next section it is useful to compare the above results with those found by Anderson and Martin (2011) who adopt a similar model and perform a similar tax and expenditure reform. The authors, following the methodology proposed by Hatta (1977), express public and private budget constraints in terms of compensated demands and the expression for the welfare change of the reform, analogous to (7) above, is in terms of the change in public expenditure $(d G)$ instead of tax changes (dt). This feature permits to discuss whether such reform is Pareto improving in terms of marginal cost of public funds and a benefit multiplier incorporating the evaluation of the public expenditure by the consumer; moreover they show the possibility to separate the decisions on tax changes from those of expenditure changes according to the information possessed by the government. With respect to their work the present paper focuses on the crucial role played by the complementarity and substitutability relations of public expenditure.

\section{Extended Model: Multiple Expenditure Categories}

The distinguishing feature of this version is the introduction of $\mathrm{M}$ categories of public expenditure, an extension that is only sketched in Anderson and Martin (2011). A vector $\mathbf{G}$ replaces the scalar $G$ of the previous section and enters as an argument into utility, demand and labour supply functions. The public budget is modified as follows:

$$
\mathbf{t x}+t_{L} L=G=\sum_{k=1}^{M} G_{k}
$$

where the scalar $G$ still represents total public expenditure, as before. Tax and expenditure reform is now characterized by a vector of tax changes and a vector of expenditure changes dG. The effect of the reform is found as before by total differentiation of indirect utility:

$$
d V=-\lambda \mathbf{x d t}+\lambda^{G} \mathbf{d G}
$$


where, quite naturally, $\lambda^{G}$ is the vector of marginal utilities associated to public expenditure categories. To simplify calculations it is assumed that, given the overall reduction in public expenditure indicated by $d G$, the required changes in different categories are given by the following shares:

$$
d G_{k}=\theta_{k} d G \text { with } \sum_{k=1}^{M} \theta_{k}=1
$$

so that $\mathbf{d G}=d G \boldsymbol{\theta}$ where $\boldsymbol{\theta}$ is the vector of the above defined shares. Total differentiation of the public budget gives:

$$
d G=\mathbf{t d} \mathbf{x}+\mathbf{x d t}+t_{L} d L
$$

Substituting into the total differential of indirect utility function yields:

$$
d V=-\lambda \mathbf{x d t}+\lambda^{G} \boldsymbol{\theta}\left(\mathbf{t d x}+\mathbf{x d t}+t_{L} d L\right)=\left(\lambda^{G} \boldsymbol{\theta}-\lambda\right) \mathbf{x d t}+\lambda^{G} \boldsymbol{\theta}\left(\mathbf{t d x}+t_{L} d L\right)
$$

By the same manipulations of the previous section we finally get:

$$
d V=\left\{\left(\lambda^{G} \boldsymbol{\theta}-\lambda\right) \mathbf{x}+\lambda^{G} \boldsymbol{\theta} \frac{\mathbf{t} \mathbf{S}+t_{L} \mathbf{L}_{i}^{C}+\left(\mathbf{t N G} \boldsymbol{\theta}+t_{L} L G \boldsymbol{\theta}-t_{L} \frac{\partial L}{\partial R}-\mathbf{t x}_{R}\right) \mathbf{x}}{\left(1-\mathbf{t N G} \boldsymbol{\theta}-t_{L} L G \boldsymbol{\theta}\right)}\right\} \mathbf{d t}
$$

where the new terms are NG and LG which are, respectively, the $\mathrm{N} \times \mathrm{M}$ matrix of expenditure effects whose representative element is:

$$
N G_{i k}=\frac{\partial x_{i}}{\partial G_{k}} \quad \mathrm{i}=1, \ldots, \mathrm{N} \quad \mathrm{k}=1, \ldots, \mathrm{M}
$$

and the vector of expenditure effects on labour supply:

$$
L G=\left(\frac{\partial L}{\partial G_{1}}, \ldots, \frac{\partial L}{\partial G_{M}}\right)
$$

Expression (15) is an immediate extension of (6) and, to ease the analysis, can be rewritten in the following more compact form:

$$
d V=\left\{A^{\prime} \mathbf{x}+\lambda_{G} \frac{B+\left(D^{\prime}-C\right) \mathbf{x}}{1-D^{\prime}}\right\} \mathbf{d t}
$$

As in the previous section, we are interested in finding sufficient conditions for a welfare gain, which is granted if both terms in curly brackets have a negative sign. An important difference between (7) and (16) has to be emphasized: all terms in (16) 
referred to public expenditure contain vector $\boldsymbol{\theta}$, which is controlled by the government, thereby giving additional degrees of freedom.

The numerator of the second term in curly brackets contains the same $B$ and $C$ as found in (7) showing substitution and income effects of the demands for private goods: $B$, in particular, suggests, as within the previous model, to concentrate tax cuts on goods with strong compensated own-price effects, small compensated (positive) cross price effects and complementary to labour.

The first term $\left(A^{\prime}\right)$ is negative if the weighted sum of marginal utilities of different expenditure categories, with weights equal to the corresponding share in overall expenditure cut, is less than marginal utility of private income: put differently, it requires that the weighted sum of marginal willingness to pay (MWP) for expenditure categories is less than one. This condition can be more easily met if available information permits to roughly partition expenditure categories according to their presumed or, better, perceived marginal utility (or MWP). Depending on the availability of such information the first term is surely negative if cuts are concentrated among categories with lower marginal utility.

Turning to the second term let's start with the denominator: $D$ ' includes all expenditure effects on private goods. A useful restriction on them is derived from the following analogue of the Slutsky equation (see also Neary and Roberts 1980, p. 33), where $\lambda_{k}$ is marginal utility of kth expenditure category:

$$
\frac{\partial x_{i}}{\partial G_{k}}=\frac{\partial h_{i}}{\partial G_{k}}+\frac{\partial x_{i}}{\partial R} \frac{\lambda_{k}}{\lambda} \quad \mathrm{k}=1, \ldots, \mathrm{M} \quad \mathrm{i}=1, \ldots, \mathrm{N}
$$

For normal goods, it establishes that if they are net substitutes (complements) for an expenditure category, substitution and income effects work in opposite (the same) direction: the reverse is true for inferior goods. This reveals a crucial role played by MWP for expenditure categories: together with (net) substitutability or complementarity relations it determines the sign of expenditure effects. Close private substitutes can be found for some expenditure categories in the fields of health and education: this means that the corresponding net substitution terms are negative; if they are sufficiently strong and, in addition, the MWP for this category is low, the income terms, which are likely to be positive, are correspondingly low so that it is presumable that the gross (aggregate) expenditure effect is negative. These circumstances make such expenditure categories natural candidates for a large cut because they contribute to a negative sign of both terms in curly brackets of expression (16) above. Nonetheless, a careful analysis of the composition of such expenditure categories is required, as suggested by a deeper reflection upon the role played by MWP; within these same categories there may exist subcategories which, though having close private substitutes, show a very large marginal utility giving rise to a gross positive effect: an example might be emergency rescue services and primary schools within the mentioned expenditures categories.

Other categories, like public works such as roads or transportation facilities, might be complements with respect to some private goods: if complement private goods are normal and their perceived utility is high they should not be cut. Nonetheless within such categories there might be sub categories highly complementary to leisure, which might be reduced. 
Finally some expenditure categories might have net substitution or complementarity relations close to zero for most private goods and labour, hence the corresponding expenditure effects is determined by income effects and MWP for such categories: if a high MWP is presumed such categories should not call for large cuts.

As a general conclusion a plurality of expenditure categories gives additional degrees of freedom: if suitable information is available on their perceived marginal utility, or marginal willingness to pay for them, it should be used to detect on which categories concentrate larger cuts. This might be sufficient to obtain a Pareto improvement if such expenditure cuts are backed by a generalised reduction in indirect tax rates. Otherwise additional information is required on the effects of indirect taxes in order to appropriately tailor tax cuts: in accordance with the conclusions of the previous section, higher tax decreases should target private goods with larger compensated own-price effects, weak relations with other private goods and complementary to labour.

The importance of the marginal willingness to pay for different expenditure categories has an important implication: it suggests to build a parallel, revised classification of expenditure categories tailored to their marginal utility and in this respect an important role could be played by the official bureau of statistics as a super partes agency vested with this particular duty. A by product of such a "revolution" might be that, within each department, a deeper attention should be paid to quality and efficiency in order to get a higher perceived utility by the citizens.

\section{Summary and Comments}

The paper analyzed the welfare effect of a combined reduction in indirect tax rates and public expenditures: its motivation originates from the current debt crisis of so many developed countries. This topic has been widely studied since the seminal contributions by Guesnerie (1977, 1995), which focused on theoretical issues of existence of directions of reform rather than on practical prescriptions for the policy maker; subsequent literature analyzed in detail theoretical conditions to make a tax and expenditure reform Pareto improving. With notable exceptions, mainly Anderson and Martin (2011), less attention was paid to modelling multiple expenditure categories: the present paper focuses on the characteristics of public expenditure categories, which make them more or less promising candidates for cuts.

The paper confirms a general result that the possibility to achieve a Pareto improvement from a combined taxes and expenditures reduction depends on information on demand for goods, labour supply and marginal utility of public expenditures or, equivalently, marginal willingness to pay for them. The bulk of information is surely burdensome but the main finding of the paper is that some degrees of freedom emerge from a more detailed description on the side of public expenditure allowing for tailoring expenditure cuts across a multiplicity of expenditure categories. In particular it is shown the importance to have reliable information on the marginal willingness to pay for different expenditure categories and in this respect an important role could be played by the central bureau of statistics to gather such information, which could be used also to partition these categories.

The paper demonstrates that a Pareto improvement requires concentrating expenditure cuts on categories with overall negative expenditure effects and tax decreases 
on goods with larger compensated own-price effects, weak relations with other private goods and complementary to labour: negative expenditure effects are found for categories with close private substitutes and a low marginal willingness to pay.

From an applied perspective the model suffers from a number of shortcomings, some of which should be mentioned at this stage; there is a single representative consumer and it is implicitly assumed a perfect mobility of workers from the public to the private sector. A natural refinement of the model should be to incorporate different consumers and types of labour or a reformulation using a social welfare function. This could properly deal with distributional issues which might arise from the required mix of expenditure cuts, given that it probably tends to favour substitute private goods with presumable regressive effects; in the real world, moreover, the implied workers mobility from the public to the private sector could take a long time to take place, with relevant distributional consequences in the meanwhile.

Nonetheless it is my opinion that even if these refinements are surely in the direction of a greater realism they should confirm the necessity to properly partition expenditure categories on the basis of reliable information concerning the marginal willingness to pay for them.

\section{References}

Ahmad E, Stern NH (1984) The theory of reform and Indian indirect taxes. J Public Econ 25:259-298

Anderson JE, Martin W (2011) Cost of taxation and benefit of public goods with multiple taxes and goods. J Public Econ Theory 13:289-309

Atkinson AB, Stiglitz JE (1980) Lectures on public economics. McGraw-Hill, New York

Auerbach AJ (1985) The theory of excess burden and optimal taxation. In: Auerbach AJ, Feldstein M (eds) Handbook of public economics, vol I. North Holland, Amsterdam

Corlett WJ, Hague DC (1953-1954) Complementarity and the excess burden of taxation. Rev Econ Stud 21:21-30

Diewert WE (1978) Optimal tax perturbations. J Public Econ 10:139-177

Guesnerie R (1977) Financing public goods with commodity taxes: the tax reform viewpoint. Econometrica 47:393-421

Guesnerie R (1995) A contribution to the pure theory of taxation. Cambridge University Press, Cambridge Hatta T (1977) The theory of piecemeal policy recommendations. Rev Econ Stud 44:1-21

Hatta T (1986) Welfare effects of changing commodity taxes rates toward uniformity. J Public Econ 29:99_ 112

Neary JP, Roberts KWS (1980) Household behaviour under rationing. Eur Econ Rev 13:25-42

Schöb R (1994) On marginal cost and marginal benefit of public funds. Public Fin 49:87-106

Slemrod J, Yitzhaki S (2001) Integrating expenditure and tax decisions: the marginal cost of funds and the marginal benefit of projects. Natl Tax J 54:189-201

Yitzhaki S, Thirsk W (1990) Welfare dominance and the design of excise taxation in Cote d'Ivoire. J Dev Econ 33:1-18 